\title{
Convergence to Nonlinear Diffusion Waves for Solutions of a System of Hyperbolic Conservation Laws with Damping
}

\author{
Ling Hsiao ${ }^{1, \star}$ and Tai-Ping $\mathrm{Liu}^{2, \star}, \star \star$ \\ 1 Institute of Mathematics, Academia Sinica, Beijing, China \\ 2 Department of Mathematics, Stanford University, Stanford, CA 94305-2125, USA
}

Received June 14, 1991

\begin{abstract}
We consider a model of hyperbolic conservation laws with damping and show that the solutions tend to those of a nonlinear parabolic equation timeasymptotically. The hyperbolic model may be viewed as isentropic Euler equations with friction term added to the momentum equation to model gas flow through a porous media. In this case our result justifies Darcy's law timeasymptotically. Our model may also be viewed as an elastic model with damping.
\end{abstract}

\section{Introduction}

Consider the following hyperbolic conservation laws with damping

$$
\begin{aligned}
v_{t}-u_{x} & =0, \\
u_{t}+p(v)_{x} & =-\alpha u, \quad \alpha>0, \quad p^{\prime}(v)<0 .
\end{aligned}
$$

The system may be viewed as isentropic Euler equations in the Lagrangian coordinates with friction term $-\alpha u$ for the momentum equation. Thus it models the compressible flow through porous media. The commonly called porous media equation is obtained by approximating the second equation with Darcy's law

$$
\begin{aligned}
v_{t} & =\frac{-1}{\alpha} p(v)_{x x}, \\
p(v)_{x} & =-\alpha u .
\end{aligned}
$$

The purpose of this paper is to show that Darcy's law may be obtained from the more complete equations (1.1) time-asymptotically. That is, solutions of (1.1) tend

* Research supported in part by Energy Dept. grant DEFG 02-88-ER25053

$\star \star$ Research supported in part by NSF grant DMS 90-0226 and Army grant DAAL 03-91-G0017 
to those of (1.2) as the time $t$ tends to infinity. In the Eulerian coordinates (1.1) and (1.2) become

$$
\begin{gathered}
\varrho_{t}+(\varrho u)_{x}=0, \\
(\varrho u)_{t}+\left(\varrho u^{2}+p(\varrho)\right)_{x}=-\alpha \varrho u, \\
\varrho_{t}=\frac{1}{\delta} p(\varrho)_{x x}, \\
p(\varrho)_{x}=-\alpha \varrho u .
\end{gathered}
$$

In this formulation both systems become degenerate at vacuum $\varrho=0$. In [2] some asymptotic analysis was carried out and particular solutions found which lead to the conjecture that the two systems are time-asymptotically equivalent even with vacuum. In the present paper we study the equivalence for the regular case away from the vacuum. System (1.1) may also be viewed as an elastic model with damping. It has been shown that the damping may prevent shock waves from forming if the initial data is not too rough [4]. As stated earlier, our interest here is not in the shock waves. Instead, we are interested in the diffusion effect the damping creates. On the other hand, even with rough data, the shock waves developed represent only transient phenomena and (1.2) would still model the time-asymptotic behavior.

In the next section we discuss self-similar solutions for (1.2) which are timeasymptotic states for general solutions. The decay estimates for such a solution will be needed in Sect. 3 where we study the behavior of solutions of (1.1) using energy estimates. We are interested in solutions of (1.1) which have limits at $x= \pm \infty$ :

$$
(u, v)(x, 0) \rightarrow\left(u_{ \pm}, v_{ \pm}\right) \quad \text { as } \quad x \rightarrow \pm \infty \text {. }
$$

Denote by any solutions of (1.2) with the same end states as $v(x, 0)$ :

$$
\begin{gathered}
\bar{v}( \pm \infty, t)=v_{ \pm}, \\
\bar{u} \equiv-\frac{1}{\delta} p(\bar{v})_{x} .
\end{gathered}
$$

Our result says that

$$
\begin{gathered}
\left\|v(x, t)-\bar{v}\left(x+x_{0}, t\right)\right\|_{L_{2}(t)}+\left\|v(x, t)-\bar{v}\left(x+x_{0}, t\right)\right\|_{L_{\infty}(t)}=O(1) t^{-1 / 2}, \\
\text { as } t \rightarrow \infty,
\end{gathered}
$$

where the translation $x_{0}$ is chosen uniquely by

$$
\int_{-\infty}^{\infty}\left(v(x, 0)-\bar{v}\left(x+x_{0}, 0\right) d x=\frac{u_{+}-u_{-}}{-\alpha} .\right.
$$

Moreover, $u \rightarrow \bar{u}$ in the following sense:

Take any smooth function $m_{0}(x)$ with compact support and

$$
\begin{gathered}
\int_{-\infty}^{\infty} m_{0}(x) d x=1 ; \\
m(x, t) \equiv-\frac{u_{+}-u_{-}}{\alpha} m_{0}(x) e^{-\alpha t}
\end{gathered}
$$


$u(x, t)$ tends to $\bar{u}(x, t)$ in the following sense:

$$
\begin{gathered}
\|(u-\bar{u}-\hat{u})(x, t)\|_{L_{2}(x)}+\|(u-\bar{u}-\hat{u})(x, t)\|_{L_{\infty}(x)}=O(1) t^{-1 / 2}, \\
\hat{u}(x, t) \equiv u_{-} e^{-\alpha t}+\int_{-\infty}^{x} m_{t}(\eta, t) d \eta, \quad \text { as } \quad t \rightarrow \infty .
\end{gathered}
$$

In the special case $v_{+}=v_{-}$we set $\bar{v}$ to be a multiple of the heat kernel:

$$
\bar{v}=v_{-}+\frac{1}{\sqrt{4 \pi t}} e^{-\frac{\left(x-p^{\prime}\left(v_{0}\right) t\right)^{2}}{4 t}} \cdot \int_{-\infty}^{\infty} v(y, 0) d y .
$$

If, further, we have $u_{+}=u_{-}$, then $m \equiv 0$. In the particular case that $\left(u_{+}, v_{+}\right)$ $=\left(u_{-}, v_{-}\right)=(0,0)$ and

$$
\int_{-\infty}^{\infty} v(y, 0) d y=0
$$

then we have $\bar{u}=\bar{v}=m=0$ and our result and the energy method used are reduced to those of [3]. The above choice of asymptotic states $\bar{v}\left(x+x_{0}\right)$ and $\bar{u}(x, t)+\hat{u}(x, t)$ is dictated by the conservation laws, cf. (3.6).

\section{Nonlinear Diffusion Equation}

It is clear that the nonlinear diffusion equation

$$
\tau_{t}=\frac{-1}{\alpha} p(\tau)_{x x}, \quad p^{\prime}(\tau)<0
$$

is invariant under $(x, t) \rightarrow\left(c x, c^{2} t\right), c>0$. Therefore, it possesses solutions of the form

$$
\tau^{*}(x, t)=\phi(x / \sqrt{t}) \equiv \phi(\xi), \quad-\infty<\xi<\infty .
$$

We are interested in solutions which have limits at $x= \pm \infty$,

$$
\phi( \pm \infty)=\tau_{ \pm}
$$

Plug (2.2) into (2.1) and integrate to obtain, for any $\xi_{0}$,

$$
\begin{aligned}
\phi^{\prime \prime}(\xi)+\frac{p^{\prime \prime}(\phi(\xi))-\frac{\alpha}{2} \xi}{p^{\prime}(\phi(\xi))} \phi^{\prime}(\xi) & =0, \\
\phi^{\prime}(\xi) & =\frac{\phi^{\prime}\left(\xi_{0}\right) p^{\prime}\left(\phi\left(\xi_{0}\right)\right)}{p^{\prime}(\phi(\xi))} e^{\zeta_{0}^{\xi} \frac{\alpha \eta}{2 p^{\prime}(\phi(\eta))} d \eta}, \\
\phi(\xi) & =\phi\left(\xi_{0}\right)+\int_{\xi_{0}}^{\xi} \frac{\phi^{\prime}\left(\xi_{0}\right) p^{\prime}\left(\phi\left(\xi_{0}\right)\right)}{p^{\prime}(\phi(\eta))} e^{\zeta_{0} \frac{\alpha s}{2 p^{\prime}(\phi(s))} d s} d \eta \\
& =\phi\left(\xi_{0}\right)+\int_{\xi_{0}}^{\xi} \phi^{\prime}(\eta) d \eta .
\end{aligned}
$$

It has been shown that (2.3) with boundary condition $(2.2)_{2}$ has a unique solution and that is strictly monotone [1]. We are also interested in the dissipative nature of 
the solution, particularly the convergence rate of $\nabla \tau^{*}$ at $x= \pm \infty$ and as $t \rightarrow \infty$. It follows from the second equation in $(2.3)$ and $p^{\prime}(\tau)<0$ that

$$
\left|\phi^{\prime}(\xi)\right| \leqq C e^{-\alpha \alpha \xi^{2}}
$$

for some $c, C>0$ independent of $\xi$. For fixed $M>0$ we have

$$
1 / C<\phi^{\prime}(\xi) / \phi^{\prime}\left(\xi_{0}\right)<C, \quad-M<\xi, \xi_{0}<M .
$$

Since

$$
\int_{-\infty}^{\infty} \phi^{\prime}(\xi) d \xi=\tau_{+}-\tau_{-}
$$

we have from the above that

$$
\left|\phi^{\prime}(\xi)\right| \leqq C\left|\tau_{+}-\tau_{-}\right| e^{-\alpha \alpha \xi^{2}} .
$$

From this and the first and last equations in (2.3) we conclude that

$$
\left|\phi^{\prime \prime}(\xi)\right|+\left|\phi^{\prime}(\xi)\right|+\left|\phi(\xi)-\tau_{+}\right|_{\xi>0}+\left|\phi(\xi)-\tau_{-}\right|_{\xi<0} \leqq c\left|\tau_{+}-\tau_{-}\right| e^{-c \alpha \xi^{2}} .
$$

From (2.1), we have

$$
\begin{aligned}
\tau_{x}^{*}(x, t) & =\frac{1}{\sqrt{t}} \phi^{\prime}(\xi), \quad \tau_{t}^{*}(x, t)=\frac{-1}{2 t} \xi \phi^{\prime}(\xi), \quad \tau_{x x}^{*}(x, t)=\frac{1}{t} \phi^{\prime}(\xi), \\
\tau_{x t}^{*}(x, t) & =\frac{1}{2 t \sqrt{t}}\left(\phi^{\prime}(\xi)+\xi \phi^{\prime \prime}(\xi)\right) .
\end{aligned}
$$

From (2.4) and (2.5) we have the following dissipative properties:

$$
\begin{gathered}
\int_{-\infty}^{\infty}\left|\tau_{x}^{*}(x, t)\right|^{2} d x=O(1)\left(\tau_{+}-\tau_{-}\right)^{2} t^{-1 / 2}, \\
\int_{-\infty}^{\infty}\left(\left|\tau_{t}^{*}(x, t)\right|^{2}+\left|\tau_{x x}^{*}(x, t)\right|^{2}\right) d x=O(1)\left(\tau_{+}-\tau_{-}\right)^{2} t^{-3 / 2}, \\
\int_{-\infty}^{\infty}\left|\tau_{x t}^{*}(x, t)\right|^{2}=O(1)\left(\tau_{+}-\tau_{-}\right)^{2} t^{-5 / 2} .
\end{gathered}
$$

\section{Asymptotic Behavior}

We want to show that the asymptotic behavior described in (1.4)-(1.9) holds for solutions of (1.1), (1.3). We set the solution $\bar{v}$ of (1.2), (1.4) to be

$$
\bar{v}(x, t) \equiv \tau^{*}(x, t+1), \quad \tau_{ \pm}=v_{ \pm},
$$

where we have avoided the singularity of $\tau^{*}(x, t)$ at $t=0$ so that $v$ satisfies (2.6)-(2.8) with $t$ replaced by $t+1$ on the right-hand side. With $m(x, t)$ given in (1.8) we set, for solution $(u, v)(x, t)$ of $(1.1),(1.3)$ and $(\bar{u}, \bar{v})$ of $(1.5)$ and $(3.1)$,

$$
\begin{gathered}
\hat{u}(x, t) \equiv u_{-} e^{-\alpha t}+\int_{-\infty}^{x} m_{t}(\eta, t) d \eta, \\
w(x, t) \equiv v(x, t)-\bar{v}\left(x+x_{0}, t\right)-m(x, t), \\
z(x, t) \equiv u(x, t)-\bar{u}\left(x+x_{0}, t\right)-\hat{u}(x, t) .
\end{gathered}
$$


We have from (1.1) and (1.5) that

$$
\begin{aligned}
w_{t}-z_{x} & =0, \\
z_{t}+[p(w+\bar{v}+m)-p(\bar{v})]_{x}+\bar{u}_{t}+\alpha z & =0 .
\end{aligned}
$$

Set

$$
y(x, t) \equiv \int_{-\infty}^{x} w(\eta, t) d \eta
$$

Note that from the conservation law $v_{t}-u_{x}=0$ in (1.1) and (1.7), (1.8) $)_{2}$,

$$
y(\infty, t)=\int_{-\infty}^{\infty} w(\eta, t) d \eta=0=y(-\infty, t)
$$

and, from (1.1) and (3.5),

$$
y_{x}=w, \quad y_{t}=\int_{-\infty}^{x} w_{t}(\eta, t) d \eta=\int_{-\infty}^{x} z_{x}(\eta, t) d \eta=z .
$$

From (3.5) and (3.6) 3 we obtain a single equation for $y$ :

$$
y_{t t}+\left[p\left(y_{x}+\bar{v}+m\right)-p(\bar{v})\right]_{x}+\alpha y_{t}-\frac{1}{\alpha} p(\bar{v})_{x t}=0 .
$$

Our basic assumption on the initial data $(u, v)(x, 0)$ of $(1.1)$ is that it tends to $\left(u_{ \pm}, v_{ \pm}\right)$ sufficiently fast as $x \rightarrow \pm \infty$ so that

$$
y(x, 0) \in H^{3}(\mathbb{R}), \quad y_{t}(x, 0) \in H^{2}(\mathbb{R}),
$$

where $H^{m}(\mathbb{R})$ are the usual Sobolev space:

$$
\|f\|_{m} \equiv \sum_{k=0}^{m}\left\|\frac{\partial^{k} f}{\partial x^{k}}\right\|_{L^{2}} .
$$

We further assume that $\left|u_{+}-u_{-}\right|+\left|v_{+}-v_{-}\right|$is small so that

$$
\left|u_{+}-u_{-}\right|+\left|v_{+}-v_{-}\right|+\|y(x, 0)\|_{3}+\left\|y_{t}(x, 0)\right\|_{2} \leqq \delta
$$

for some small $\delta$. We will show that $y(x, t)$ also satisfies the same smallness condition as in (3.8). By the Sobolev lemma we have

$$
|f|_{c^{k}} \leqq C\|f\|_{k+1} \text {. }
$$

Thus in the following energy estimate we will make the a-priori hypothesis

$$
|y(x, t)|_{c^{2}(x)}+\left|y_{t}(x, t)\right|_{c^{1}(x)} \leqq C \delta
$$

for some $C$ independent of $t$. Multiply (3.7) by $y$ and integrate to obtain

$$
\begin{aligned}
& \left.\int_{-\infty}^{\infty} \alpha \frac{y^{2}}{2} d x\right|_{0} ^{T}+\left.\int_{-\infty}^{\infty} y y_{t} d x\right|_{0} ^{T}-\int_{0}^{T} \int_{-\infty}^{\infty} y_{t}^{2} d x d t \\
& -\int_{0}^{T} \int_{-\infty}^{\infty} y_{x}\left[p\left(y_{x}+\bar{v}+m\right)-p(\bar{v})\right] d x d t+\int_{0}^{T} \int_{-\infty}^{\infty} \frac{1}{\alpha} y_{x} p(\bar{v})_{t} d x d t=0 .
\end{aligned}
$$


Since $p^{\prime}<0$, and $\bar{v}$ satisfies (2.6)-(2.8) and that $m$ satisfies (1.8), we have, for $\delta$ small, that the above yields

$$
\begin{aligned}
& \int_{-\infty}^{\infty} y^{2}(x, T) d x+\int_{0}^{T} \int_{-\infty}^{\infty} y_{x}^{2} d x d t \\
& =O(1)\left[\delta^{2}+\int_{-\infty}^{\infty} y_{t}^{2}(x, T) d x+\int_{0}^{T} \int_{-\infty}^{\infty} y_{t}^{2} d x d t\right] .
\end{aligned}
$$

Here we have used the Cauchy-Schwarz inequality.

Multiply (3.7) by $y_{t}$ and integrate to obtain

$$
\begin{aligned}
& \left.\frac{1}{2} \int_{-\infty}^{\infty} y_{t}^{2} d x\right|_{0} ^{T}+\int_{0}^{T} \int_{-\infty}^{\infty} \alpha y_{t}^{2} d x d t-\int_{0}^{T} \int_{-\infty}^{\infty}\left[p\left(y_{x}+\bar{v}+m\right)-p(\bar{v})\right] y_{x t} d x d t \\
& -\int_{0}^{T} \int_{-\infty}^{\infty} \frac{1}{\alpha} p(\bar{v})_{x t} y_{t} d x d t=0 .
\end{aligned}
$$

Set

$$
\begin{gathered}
q(x, t) \equiv-\int_{0}^{y_{x}}[p(\eta+\bar{v}(x, t))-p(\bar{v}(x, t))] d \eta \\
-q_{t}=\left[p\left(y_{x}+\bar{v}\right)-p(\bar{v})\right] y_{x t}+\int_{0}^{y_{x}}\left[p^{\prime}(\eta+\bar{v})-p^{\prime}(\bar{v})\right] \bar{v}_{t} d \eta .
\end{gathered}
$$

Since $p^{\prime}<0$ we have

$$
C_{2}\left(y_{x}\right)^{2} \leqq q \leqq C_{1}\left(y_{x}\right)^{2}
$$

for some $C_{1}, C_{2}>0$. We have from above and (2.7), (2.8) for $\bar{u}$ that

$$
\begin{aligned}
- & \int_{0}^{T} \int_{-\infty}^{\infty}\left[p\left(y_{x}+\bar{v}\right)-p(\bar{v})\right] y_{x t} d x d t \\
& =\left.\int_{-\infty}^{\infty} q d x\right|_{0} ^{T}+\int_{0}^{T} \int_{-\infty}^{\infty} \int_{0}^{y_{x}}\left[p^{\prime}(\eta+\bar{v})-p^{\prime}(\bar{v})\right] \bar{v}_{t} d \eta d x d t \\
& =\left.\int_{-\infty}^{\infty} q d x\right|_{0} ^{T}+\int_{0}^{T} \int_{-\infty}^{\infty} O(1) y_{x}^{2} \bar{v}_{t} d x d t \\
& =\left.\int_{-\infty}^{\infty} q d x\right|_{0} ^{T}+O(1) \delta \int_{-\infty}^{\infty} y_{x}^{2} d x d t .
\end{aligned}
$$

Thus (3.11), the above and (1.8), (3.9) yield

$$
\int_{-\infty}^{\infty}\left(y_{t}^{2}+y_{x}^{2}\right)(x, T) d x+\int_{0}^{T} \int_{-\infty}^{\infty} y_{t}^{2} d x d t=O(1) \delta^{2}+O(1) \delta \int_{0}^{T} \int_{-\infty}^{\infty} y_{x}^{2} d x d t .
$$

We have from this and (3.10) the basic estimate

$$
\int_{-\infty}^{\infty}\left(y^{2}+y_{t}^{2}+y_{x}^{2}\right)(x, T) d x+\int_{0}^{T} \int_{-\infty}^{\infty}\left(y_{x}^{2}+y_{t}^{2}\right) d x d t=O(1) \delta^{2},
$$

where we have used the smallness of $\delta$.

Instead of integrating $(3.7) \cdot y_{t}$ over $0 \leqq t \leqq T,-\infty<x<\infty$, to obtain (3.11) we integrate it over $(t, T) \times(-\infty, \infty)$ to obtain

$$
\begin{aligned}
\int_{-\infty}^{\infty}\left(\frac{1}{2} y_{t}^{2}+q\right)(x, T) d x \leqq & \int_{-\infty}^{\infty}\left[\frac{1}{2} y_{t}^{2}+q\right](x, t) d t \\
& +\int_{t}^{T} \int_{-\infty}^{\infty}\left[O(1) y_{x}^{2} \bar{v}_{t}+O(1) p(\bar{v})_{x t}^{2}+O(1) m^{2}\right] d x d t .
\end{aligned}
$$


We then integrate the above for $0 \leqq t \leqq T$ :

$$
\begin{aligned}
T & \int_{-\infty}^{\infty}\left(\frac{1}{2} y_{t}^{2}+q\right)(x, T) d x \leqq \int_{0}^{T} \int_{-\infty}^{\infty}\left[\frac{1}{2} y_{t}^{2}+q\right] d x d t \\
& +\int_{0}^{T} d s \int_{s}^{T} \int_{-\infty}^{\infty}\left[O(1) y_{x}^{2} \bar{v}_{t}+O(1) p(\bar{v})_{x t}^{2}+O(1) m^{2}\right] d x d t \\
& =\int_{0}^{T} \int_{-\infty}^{\infty}\left[\frac{1}{2} y_{t}^{2}+q\right] d x d t+\int_{0}^{T} \int_{-\infty}^{\infty} t\left[O(1) y_{x}^{2} \bar{v}_{t}+O(1) p(\bar{v})_{x t}^{2}+O(1) m^{2}\right] d x d t .
\end{aligned}
$$

From the estimates (2.6)-(2.8), (3.1) for $\bar{u},(1.8)$ for $m$, and (3.12), (3.13) we have from the above

$$
T \int_{-\infty}^{\infty}\left(y_{t}^{2}+y_{x}^{2}\right)(x, T) d x=O(1) \delta^{2} .
$$

This and (3.13) give rise to the basic decay estimate:

$$
\int_{-\infty}^{\infty}\left(y_{t}^{2}+y_{x}^{2}\right)(x, T) d x=O(1) \delta^{2}(T+1)^{-1} .
$$

From (3.3), (3.4) and (3.6) $)_{3}$ this becomes

$$
\|(u-\bar{u}-\hat{u})(\cdot, t)\|_{L^{2}}+\|(v-\bar{v}-m)(\cdot, t)\|_{L^{2}}=O(1) \delta(t+1)^{-1 / 2} .
$$

Similar arguments as above we have, based on (3.8) and (3.9), that

$$
\|y(x, t)\|_{3}+\left\|y_{t}(x, t)\right\|_{2} \leqq C \delta,
$$

and the solution to (1.1), (1.2) exists. The decay estimate (3.15) can also be generalized to higher derivatives. In particular, we have

$$
\|(u-\bar{u}-\hat{u})(x, t)\|_{H^{1}}+\|(v-\bar{v}-m)(x, t)\|_{H^{1}}=O(1) \delta(t+1)^{-1 / 2} .
$$

From (3.16) we have from the Sobolev lemma the pointwise decay

$$
\sup _{x}(|u-\bar{u}-\hat{u}|(x, t)+|v-\bar{v}-m|(x, t))=O(1) \delta(t+1)^{-1 / 2} .
$$

This proves (1.6) and (1.9).

\section{References}

1. Duyn, C.T. Van Peletier, L.A.: Nonlinear analysis. T.M.A. 1, 223-233 (1977)

2. Liu, T.-P.: Nonlinear hyperbolic-parabolic partial differential equations. Nonlinear Analysis, Proceedings, 1989 Conference. Liu, F.C., Liu, T.P. (eds.), pp. 161-170. Academia Sinica, Taipei, R.O.C.: World Scientific

3. Matzumura, A.: Nonlinear hyperbolic equations and related topics in fluid dynamics. Nishida, T. (ed.). Pub. Math. D'Orsay, 53-57 (1978)

4. Nishida, T.: Nonlinear hyperbolic equations and related topics in fluid dynamics. Nishida, T. (ed.). Pub. Math. D’Orsay, 46-53 (1978) 
